\title{
Ideas sobre la vejez en estudiantes de Ciencias de la Salud del Centro Universitario de Tonalá
}

Wilfrido Domínguez Sandoval, ${ }^{1}$ Mónica Carolina Estrada Galindo, ${ }^{1}$ Diana Gisell Ibarra Figueroa, ${ }^{1}$ Melissa Sarahí Villalobos Pulido ${ }^{1}$ y Neyda Ma. Mendoza Ruvalcaba ${ }^{1}$

\section{Introducción}

Actualmente, la población mexicana transita por un envejecimiento demográfico de moderado a avanzado (Villagómez, 2010) que recientemente ha sido intitulado como "envejecimiento generacional", en el que el número de adultos mayores será mayor a la población de infantes.

Algunos estudios demuestran que entre los años 2000 y 2050 la población de los adultos mayores pasará de 7\% a 28\% en una población de 130 millones para el año 2050 (Franco, Villarreal, Vargas, Martínez y Galicia, 2010). A la par de esta transición sociodemográfica, se prevé una transición epidemiológica, debido a que una mayor prevalencia de enfermedades crónico-degenerativas se encuentra asociada a la edad, ello podría tener un gran impacto en los ámbitos de atención social y sobre los servicios de salud.

Numerosos estudios evidencian la gran discriminación existente en el área de la salud hacia los adultos mayores, sobre

1 Centro Universitario de Tonalá, Universidad de Guadalajara. 
todo por parte de los profesionales (Williams, 2009). Es importante resaltar que uno de los primeros problemas se gesta durante la formación académica al no tener conocimientos generalizados, en especial al no contar con la información adecuada sobre determinados grupos poblacionales, concretamente sobre las personas mayores; ello puede constituir el inicio de un prejuicio que potencialmente perjudica en la interacción durante la práctica profesional y en la formación de actitudes que pueden ser interpretadas como discriminativas (Caruthers, 1998).

En este sentido, se ha encontrado que cuando se trata de brindar atención a diferentes cohortes, no se utilizan con personas mayores los mismos tratamientos que normalmente se aplicarían a pacientes con una edad cronológica más corta. Se ha evidenciado, además, el maltrato hacia los pacientes geriátricos, la utilización de nombres despectivos y un ambiente hostil (Williams, 2009). Asimismo, se ha demostrado que la población en general y especialmente los profesionales de la salud desconocen y tienen ideas falsas de aspectos relevantes al envejecimiento (Seufert y Carrozza, 2002). El concepto y actitudes de los estudiantes de las Ciencias de la Salud frente a la vejez se construyen, como en la mayoría de la población, gracias a las ideas socialmente vigentes.

El presente trabajo tiene el objetivo de analizar y comparar los estereotipos acerca de las personas mayores por parte de estudiantes de Ciencias de la Salud, específicamente de Gerontología, Salud Pública y Medicina.

\section{Método}

Estudio transversal analítico de metodología cuantitativa

Se entrevistó a 104 estudiantes de ambos sexos del Centro Universitario de Tonalá de la Universidad de Guadalajara del área de Ciencias de la Salud de tercer y cuarto semestre $(\mathrm{n}=$ 35 Gerontología, $n=38$ Medicina, $n=31$ Salud Pública), de los cuales $68.3 \%$ fueron mujeres y $31.7 \%$ hombres con una edad 
promedio de 21.3 (DS 4.7) años con un rango mínimo de 19 y un máximo de 58 años.

Para la recolección de datos se empleó el Cuestionario de Estereotipos hacia la Vejez de Montorio e Izal en la versión propuesta por Mena, Sánchez y Trianes (2005): Cuestionario de Estereotipos Negativos hacia la Vejez (CENVE), el cual está compuesto por 13 ítems y tres factores referidos a las siguientes dimensiones:

1. Salud: afirmaciones acerca del deterioro general de ésta y de las discapacidades asociadas.

2. Motivación-social: creencias acerca de las carencias afectivas y sociales.

3. Carácter-personalidad: rigidez mental y problemas de labilidad emocional.

Cada ítem está medido con una escala Likert del 1 al 4 (categoría: 1 = muy de acuerdo, 2 = de acuerdo, $3=$ en desacuerdo, 4 = muy en desacuerdo), donde las puntuaciones altas indican un elevado grado de creencias en los estereotipos negativos de la vejez, mientras puntuaciones bajas indican poco nivel de creencia en tales estereotipos negativos. Adicionalmente se recabaron datos sociodemográficos.

Los estudiantes fueron informados acerca del estudio y aceptaron participar de manera voluntaria. Se elaboró una base de datos electrónica donde se capturaron y se validaron los datos. Los resultados se analizaron mediante el programa spss (versión 20). Se calcularon promedios y comparación de proporciones por medio de la prueba de chi-cuadrada y se compararon las medias estadísticas por medio de análisis de ANOVA, con una $p>.05$.

\section{Resultados}

Después del análisis de datos se encontraron los siguientes hallazgos. 


\section{Factor salud}

En el ítem "fuerte deterioro de la salud" se encontró que los estudiantes de Medicina cuentan con una visión mayormente negativa (media $=2.82$, DS $=0.56$ ), seguido por la licenciatura en Salud Pública con una pequeña diferencia (media $=2.41$, DS $=$ 0.61 ); los estudiantes de Gerontología (media $=1.89, \mathrm{DS}=0.67$ ) son aquellos que cuentan con una visión más positiva en este factor $(p<=0.000)$. Entre Medicina y Salud Pública no se encontraron diferencias significativas.

Dentro del mismo factor se encontró, respecto a la idea de que las personas adultas mayores "tienen más incapacidades por las que dependen de los demás", que los estudiantes de Salud Pública (media $=2.61$, DS $=0.71$ ) y los de Medicina (media $=2.53$, DS $=0.76$ ) reportan un estereotipo mayormente negativo $(p<=0.000)$, a diferencia de los estudiantes de Gerontología (media $=1.49$, DS $=0.50$ ). Entre los tres grupos se evidencia una similitud considerable, específicamente entre los estudiantes de Salud Pública y Medicina.

Respecto a la idea de que hay mayor "deterioro de la memoria" se encontró que aquellos que presentan estereotipos más negativos son los estudiantes de Salud Pública (media $=2.65$ ) (DS $=0.75$ ), seguidos por los de Medicina (media $=2.55$, DS = 0.64). Finalmente, se encuentran los estudiantes de Gerontología (media $=1.71$, DS $=0.66$ ), quienes muestran el menor grado de estereotipos hacia la vejez $(p<=0.000)$.

\section{Factor motivacional-social}

Respecto al ítem "tienen menos amigos que las personas más jóvenes", los estudiantes de Medicina (media=2.26, DS=0.92) presentaron una visión mayormente negativa hacia la vejez $(p<$ $=0.000$ ), a diferencia de los estudiantes de Salud Pública (media $=1.94, \mathrm{DS}=0.89$ ) y Gerontología (media $=1.57, \mathrm{DS}=0.60$ ). Dentro de los siguientes cuatro ítems del factor motivacional-social se encontró una diferencia de $p<=0.000$ estadísticamente significativa en cada ítem respectivamente. Para el ítem "las per- 
sonas mayores tienen menos interés por el sexo", las percepciones más negativas son para los estudiantes en Medicina (media $=2.61$, DS $=0.59$ ) y Salud Pública (media $=2.59$, DS = 0.90), en comparación con los estudiantes en Gerontología (media = 1.74, DS = 0.65). Asimismo, el mayor grado de estereotipos negativos hacia la vejez respecto al ítem "Incapaces de resolver problemas" se registró en los estudiantes de Medicina (media = $2.11, \mathrm{DS}=0.68$ ) y Salud Pública (media $=2.10$, DS $=0.83$ ), y en menor medida en los de Gerontología (media $=1.46, \mathrm{SD}=0.56$ ) $(p<=.000)$.

Con relación a la idea de que las personas mayores tienen "pérdida de interés por las cosas", las percepciones más negativas corresponden de manera similar a los estudiantes de Medicina y Salud Pública, el valor de las medias fueron de 2.18 (DS $=0.75)$ y $2.32(\mathrm{DS}=0.74)$ respectivamente; en relación con los estudiantes de Gerontología se encontró una media de 1.51 (DS $=0.56$ ), la cual indica un menor grado de estereotipos a diferencia del resto de los participantes.

Sobre la creencia "no realizan el trabajo tan bien como los jóvenes", la idea más negativa corresponde a los estudiantes de Salud Pública (media $=2.81$, DS $=0.91$ ), seguido por los estudiantes de Medicina, con una media de 2.32 (DS $=0.80$ ) con una diferencia significativa entre ambos $(p<=0.000)$. Los estudiantes en Gerontología (media $=1.46$, DS $=0.61$ ) presentan un grado menor de estereotipos negativos en este ítem. Es importante enfatizar que el ítem que muestra mayor grado de estereotipo dentro de los tres grupos de estudiantes es "las personas mayores tienen menos interés por el sexo".

\section{Factor carácter-personalidad}

En relación con la idea "se vuelven más rígidas e inflexibles" se encontró una visión mayormente negativa en estudiantes de Medicina $($ media $=2.95, \mathrm{DS}=0.73)$ y Salud Pública $($ media $=$ 2.84 , DS $=0.77$ ), en comparación con los estudiantes de Gerontología (media $=1.71, \mathrm{DS}=0.57$ ), quienes presentan menor estereotipo hacia la vejez. Con respecto a la idea "Son como 
niños", los estudiantes de Medicina son aquellos que presentan un estereotipo mayormente negativo (media $=3.24$, DS $=0.59$ ), seguidos de los estudiantes de Salud Pública (media $=2.90$, DS $=0.74$ ) y Gerontología (media $=1.54$, DS $=0.65$ ). La percepción sobre "Los defectos se agudizan con la edad" resulta significativamente más negativa en estudiantes de Salud Pública (media = 2.47, DS $=0.88$ ), seguido de los estudiantes de Medicina (media $=2.42$, DS $=0.82$ ) y Gerontología (media $=1.86$, DS $=0.87$ ). La creencia en relación con "se irritan y son cascarrabias" resulta ser mayormente negativa en los estudiantes de Salud Pública (media $=2.74$, DS $=0.81$ ), enseguida los estudiantes de Medicina (media=2.47, DS=0.76) y Gerontología (media $=1.49$, DS $=0.61$ ). Finalmente la idea "chochean" fue mejor aceptada por estudiantes de Medicina (media $=2.74$, DS $=0.79$ ), Salud Pública (media $=2.61$, DS $=0.66$ ) y finalmente Gerontología (media $=1.54, \mathrm{DS}=0.61)$.

\section{Conclusiones}

Podemos concluir que los estudiantes de Ciencias de la Salud tienen estereotipos o ideas negativas hacia la vejez. En particular, los estudiantes de Medicina y Salud Pública reportan una percepción mayormente negativa en las tres dimensiones: salud, motivacional-social y carácter-personalidad; a diferencia de los estudiantes de Gerontología, lo cual es esperado debido a que en la formación de estos últimos se incluye constantemente la promoción de actitudes positivas así como la habilidad para identificar y eliminar ideas negativas hacia la vejez.

En general, las ideas significativamente más negativas fueron: "tienen un fuerte deterioro de la salud", "no realizan un trabajo tan bien como los jóvenes", "se vuelven más rígidos e inflexibles", "son como niños", "se irritan y son cascarrabias", y "chochean". Específicamente, en la dimensión carácter-personalidad, las ideas son más negativas con respecto a los adultos mayores en cuanto a que "son como niños" y con menor grado a la idea de que "los defectos se agudizan con la edad". En las 
licenciaturas de Medicina y Salud Pública se encontró el mismo estereotipo. Los estudiantes de Gerontología estereotipan con mayor grado la idea de que "los defectos se agudizan con la edad", y con menor medida la idea de que "se irritan y son cascarrabias".

Los prejuicios hacia la vejez denotan la falta de una visión realista por parte de los estudiantes y representa un área de desarrollo que se debería reforzar desde los programas académicos. Es fundamental sensibilizar y promover en los nuevos profesionales de la salud un enfoque real acerca de la vejez y el adulto mayor, para evitar la discriminación y los prejuicios en la práctica profesional desde cada disciplina.

\section{Referencias}

Caruthers, C. (1998). Ageism in the Preclinical Years. Journal of the American Medical Association, 279(13), 1035.

Franco, M., Villarreal, E., Vargas, E., Martínez, L., Galicia, L. (2010). Estereotipos negativos de la vejez en personal de salud de un Hospital de la Ciudad de Querétaro, México. Revista Médica de Chile, 138(8), 988-993.

Mena, M., Sánchez, C., Trianes, M. (2005). Cuestionario de evaluación de estereotipos negativos hacia la vejez. Revista Multidisciplinar de Gerontología, 5(4), 212-220.

Seufert, R., Carrozza, M. (2002). A test of Palmore's Facts on Aging Quizzes as Alternate Measures. Journal of Aging Studies, 16, 279-294.

Villagómez, P. (2010). El envejecimiento demográfico en México: niveles, tendencias y reflexiones en torno a la población de adultos mayores. En L. Gutiérrez y J. Gutiérrez (Coord.), Envejecimiento humano, una visión transdisciplinaria (305314). México: Secretaría de Salud, Instituto de Geriatría.

Williams, P. (2009). Age Discrimination in the Delivery of Health Care Services to Our Elders. Marquette Elder's Advisor, 11(1), 1-46. 
